\title{
Synapses seen at different scales
}

\begin{abstract}
Synapse morphology and molecular composition can be analyzed in detail with conjugate light-electron array tomography, which combines immunofluorescence imaging with electron microscopy in a precisely aligned fashion.
\end{abstract}

Synapses vary in their morphology and composition, depending on the cell types that they connect and their function in a given circuit. Whereas electron microscopy (EM) provides structural information, the molecular composition of synapses is more readily addressed with immunofluorescence imaging approaches. Forrest Collman and Stephen Smith from the Allen Institute for Brain Science, in collaboration with Richard Weinberg from the University of North Carolina, used conjugate light-electron array tomography to analyze the molecular composition of individual synapses in the mouse cortex.

Array tomography involves the preparation of arrays of serial ultrathin tissue sections, imaging of the arrays with electron or immunofluorescence light microscopy techniques, and reconstruction and overlay of the images in a precisely aligned fashion. Combining the different imaging modalities allows the data, which are obtained at different scales, to be correlated. For studying synapses, these correlative approaches provide single-synapse resolution as well as details on the molecular composition of these synapses, which is not possible with the individual methods on their own.

Collman considers conjugate lightelectron array tomography a special case in the canon of correlative methods. "The word 'correlative'... has come to embody an extremely broad set of methods," says Collman. In their paper, Collman and his colleagues performed immunofluorescence and electron microscopy on the same ultrathin sections rather than on adjacent sections or even different samples as in other correlative approaches. Although it is desirable to obtain images from the same sec-

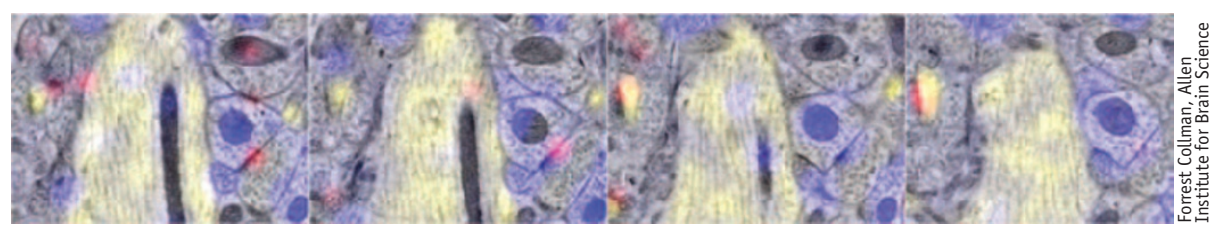

Consecutive scanning electron micrographs of a region in the mouse neocortex, overlaid with the corresponding immunofluorescence images.

tion, the need to preserve both the epitopes for immunofluorescence microscopy and the structure for EM poses a challenge. Usually, "there is a trade-off between getting good immunofluorescence and good EM ultrastructure," explains Collman.

To overcome the sample-preparation hurdle and to be able to generate highquality data in both imaging modalities, Collman and Smith combined their imaging and array expertise with Weinberg's tissue preparation expertise. They established that both ultrastructure and immunogenicity are well preserved when the tissue samples are fixed with a mixture of aldehydes and embedded in the plastic resin Lowicryl without further osmium treatment, which is otherwise commonly used in EM preparations.

Arrays generated from tissues prepared in such a way can be subjected to multiple rounds of immunofluorescence imaging by eluting the antibodies used in between the consecutive rounds of imaging. Typically, Collman and his colleagues analyze the distribution of up to nine different proteins in three rounds of imaging, which are followed by scanning EM analysis of the sections. "I don't think that we have fully characterized what the upper limit is," says Collman, regarding the level of antibody multiplexing that may be possible. He concedes that image contrast in the EM data is reduced by the immunostaining protocol, possibly owing to the elution procedure required for antibody multiplexing, but he thinks that the number of rounds does not have a strong influence on the outcome. "The biggest hit is definitively the first time that [the sample] is exposed to the elution solution. The difference of one round versus three rounds is much more subtle," he says.

The researchers applied their methodology to analyze the molecular composition of synapse subtypes in the mouse neocortex, where synapses of different function are intermingled. This complexity and diversity requires analysis methods with single-synapse resolution, such as conjugate light-electron array tomography. Neurons in this brain region generally use either glutamate or GABA ( $\gamma$-aminobutyric acid) as neurotransmitters and can themselves receive either glutamatergic or GABAergic input. Using a panel of antibodies against pre- and postsynaptic proteins, the researchers could classify the synapses they identified in the EM data set into the expected four subtypes, on the basis of their protein composition. This classification was possible even using an automated algorithm.

In the future, Collman wants to apply conjugate light-electron array tomography to study better-defined synapse populations, combining analyses of synaptic function with the underlying structure. "In the paper, we were looking at the molecular composition of ... pretty broad classes of both presynaptic and postsynaptic targets. We want to be looking at much more specific populations of synapses than that," he concludes. Nina Vogt

\section{RESEARCH PAPERS}

Collman, F. et al. Mapping synapses by conjugate light-electron array tomography. J. Neurosci. 35, 5792-5807 (2015). 